\title{
Elaboração e validação de tabela MNREAD para o idioma português
}

\section{Development and validation of the MNREAD reading acuity chart in Portuguese}

\author{
Celina Tamaki Monteiro de Castro ${ }^{1}$ \\ Christopher Scott Kallie ${ }^{2}$ \\ Solange Rios Salomão ${ }^{3}$
}

Trabalho realizado no Departamento de Oftalmologia da Universidade Federal de São Paulo (UNIFESP).

${ }^{1}$ Pós-Graduanda do Programa de Doutorado em Ciências Visuais do Departamento de Oftalmologia da Universidade Federal de São Paulo (UNIFESP). São Paulo (SP). Brasil.

${ }^{2}$ B.A. Psychology, University of Minnesota, Minnesota Laboratory for Low-Vision Research. Minneapolis (MN). USA.

Professora Associada Livre Docente do Departamento de Oftalmologia da UNIFESP. São Paulo (SP). Brasil.

Endereço para correspondência: Celina Tamaki M. Castro - Rua Botucatu, 822 - São Paulo (SP). Brasil. CEP 04023-062

E-mail: celina@oftalmo.epm.br

Recebido para publicação em 15.09.2004

Versão revisada recebida em 10.03.2005

Aprovação em 15.06.2005

Nota Editorial: Depois de concluída a análise do artigo sob sigilo editorial e com a anuência da Dra. Keila Miriam Monteiro de Carvalho sobre a divulgação de seu nome como revisora, agradecemos sua participação neste processo.

\section{RESUMO}

Objetivos: Construir e validar a tabela "Minnesota Low Vision Reading Test" - MNREAD na versão do idioma Português. Métodos: A tabela de acuidade e leitura do "Minnesota Low Vision Reading Test" contém 19 sentenças (logMAR 0,5 a 1,3) com 60 caracteres impressos em três linhas. Todas as sentenças devem possuir o mesmo comprimento e vocabulário simples. Foi construído um total de 110 sentenças. As sentenças foram testadas em 36 sujeitos (20 adultos e 16 crianças); o tempo de leitura e os erros cometidos foram apurados e a média de velocidade de leitura e os erros foram analisados. Trinta e oito sentenças foram selecionadas para a construção do protótipo(MNREAD-P). Foram excluídas sentenças com alta e baixa média de velocidade de leitura, alto desvio padrão e sentenças com erros repetidos cometidos pelos sujeitos. Validação: Vinte sujeitos com visão normal ( $\log$ MAR 0 ou mais, com a melhor correção) foram testados na tabela MNREAD-P e leram um texto de leitura do dia-a-dia. A velocidade de leitura em palavras/minuto foi medida na tabela e no texto. Resultados: As sentenças da tabela MNREAD-P são suficientemente consistentes para medidas confiáveis da habilidade de leitura. A velocidade de leitura do texto ( $\log$ MAR $=0,6$ ) foi 197,8 palavras/minuto e a velocidade máxima na tabela MNREAD-P foi 200,1 palavras/minuto. A correlação entre as duas medidas foi $r=0,82$. Conclusões: A tabela MNREAD-P foi testada em sujeitos com visão normal e os resultados foram os mesmos da "Minnesota Low Vision Reading Test" original e a velocidade de leitura foi estatisticamente equivalente à da velocidade de leitura do texto.

Descritores: Acuidade visual; Visão; Leitura; Baixa visão/fisiopatologia; Psicofísica Testes de visão; Valor preditivo dos testes; Validação de programas de computador

\section{INTRODUÇÃ̃O}

A leitura tem importante papel nas interações sociais e nas atividades produtivas. Medidas convencionais da função visual, como a acuidade visual obtida na tabela de Snellen, têm valor limitado para refletir o desempenho nas tarefas do dia-a-dia, principalmente em indivíduos com deficiência visual ${ }^{(1-2)}$. A habilidade de leitura vem se tornando um padrão de medida da visão funcional ${ }^{(3)}$, o que provocou o desenvolvimento de testes de acuidade de leitura como a tabela de Sloan $\mathrm{M}^{(4)}$, a tabela de leitura de palavras Bailey-Lovie ${ }^{(5)}$, o teste Pepper ${ }^{(6)}$ e a tabela Minnesota Low Vision Reading Test - MNREAD (teste de acuidade de leitura Minnesota) ${ }^{(7-8)}$.

A leitura é uma das atividades da vida diária mais prejudicadas em pacientes com visão baixa. Muitos pacientes com degeneração macular relacionada à idade, alfabetizados antes da diminuição da visão, conside- 
ram-se "analfabetos" após a doença ${ }^{(9)}$. A incapacidade de ler jornal com a melhor correção óptica, numa distância normal de leitura é às vezes usada como definição de visão baixa ${ }^{(1)}$, portanto, testes clínicos de leitura apropriados devem ser aplicados ${ }^{(7)}$ e a prescrição de auxílios ópticos para leitura não deve ser baseada somente na acuidade visual de perto e sim na fluência de leitura de texto ${ }^{(10)}$.

O "Minnesota Low Vision Reading Test" - MNREAD é um teste de leitura que foi especialmente desenhado para ser sensível aos fatores visuais. A tabela impressa MNREAD oferece uma maneira rápida, simples e confiável de testar a acuidade e velocidade de leitura de indivíduos com visão normal e visão baixa. As medidas de velocidade de leitura são inseridas no gráfico da tabela MNREAD e automaticamente, dados como velocidade de leitura (palavras/minuto), tempo levado para a leitura (segundos) e o tamanho da letra (logMAR) lidos são facilmente visualizados.

Três tabelas baseadas na versão americana do teste MNREAD já foram desenvolvidas em outros idiomas: italiano, japonês e francês. O desenvolvimento de versões em outros idiomas da tabela MNREAD seguiu todas as etapas do protocolo internacional MNREAD*, que estabelece alguns princípios de implementação baseados na tabela americana. Cada versão em outro idioma precisa ser criada e desenvolvida, pois a tabela não pode ser construída utilizando-se uma simples tradução da tabela americana existente.

O objetivo deste estudo foi a elaboração e validação de versão da tabela MNREAD para o idioma português (MNREAD-P).

\section{MÉTODOS}

Foram testados 36 indivíduos (20 adultos e 16 crianças) com idades variando de 8 a 47 anos no protótipo da tabela MNREAD-P. O protocolo de estudo foi analisado e aprovado pelo Comitê de Ética em Pesquisa da UNIFESP. Os critérios de inclusão no estudo foram ter o idioma português como primeira língua, acuidade visual com a melhor correção óptica para longe e para perto de 20/20 em cada olho, medida com a tabela ETDRS a 4 m e com a tabela "Lighthouse Near Visual Acuity Test" a $40 \mathrm{~cm}$, ausência de alterações de fundo de olho, ausência de problemas de leitura e concessão do termo de consentimento livre e esclarecido por parte do sujeito ou seu responsável. Todos os testes foram feitos em condição binocular na distância de $40 \mathrm{~cm}$, com iluminação padronizada de 80 candelas $/ \mathrm{m}^{2}$. A tabela MNREAD possibilita as seguintes medidas: a) acuidade de leitura: a menor letra que o paciente consegue ler sem cometer erros significantes; b) tamanho crítico de letra: tamanho mínimo de letra que o paciente consegue ler com máxima velocidade e c) velocidade máxima de leitura: velocidade de leitura do paciente quando a leitura não está limitada pelo tamanho da letra.

\footnotetext{
* MNREAD 2000 project [tabela]. Minnesota Laboratory for Low-vision Research. Minneapolis, MN; University of Minnesota, Department of Psychology; 2002. [cited 2005 Mar 19]. Available from: http://gandalf.psych.umn.edu/ gellab/MNREAD/ MNREAD2000
}

\section{Elaboração de protótipo da tabela MNREAD para o idioma português}

As frases que compõe a tabela de acuidade e velocidade de leitura na MNREAD-P, foram elaboradas seguindo-se o seguinte critério da tabela MNREAD americana: todas as frases têm que ter o mesmo comprimento e 60 caracteres distribuídos em 3 linhas que se encaixam em uma caixa retangular de aspecto proporcional fixo (Figura 1). No processo de criação das sentenças utilizou-se o "mntest"**, um aplicativo simples em "javascript" que testou as frases quanto ao tamanho de cada linha e quanto ao número de caracteres (Figura 2).

Antes de iniciar a construção das frases que compõem a tabela, foram pesquisadas palavras de vocabulário simples mais comumente usadas por crianças das $2^{\underline{a}}$ e $3^{\underline{a}}$ séries do ensino fundamental e a escolha foi realizada baseada em livros infantis e em estudos da área de lingüística ${ }^{(11-14)}$.

Um teste piloto foi realizado após elaboradas as primeiras 59 sentenças, que obedeceram aos critérios de construção descritos acima, e um teste de leitura dessas sentenças foi realizado em 10 sujeitos adultos, sendo 7 do sexo masculino e 3 do sexo feminino, com idade variando de 19 a 45 anos. Nenhum sujeito relatou dificuldades na leitura. Os participan-

\section{O rapaz entrega sua mercadoria todas as manhãs de bicicleta}

Figura 1 - Modelo de frase testada para confecção da tabela MNREAD, composta de 60 caracteres distribuídos em 3 linhas

\begin{tabular}{|cccc|}
\hline text & chars & width & \\
As_janelas_da_nossa_ & 20 & 00.9985 & ok \\
casa_estao_pintadas_ & 20 & 00.9736 & ok \\
de_cinza_e_vermelho_ & 20 & 1.027 & ok \\
Character count 60 & \multicolumn{2}{c}{ ok } & \\
clear & check sentence & \\
& &
\end{tabular}

Figura 2 - Exemplo de teste de frase para a tabela MNREAD-P, após ser analisada pelo aplicativo "mntest", em que o comprimento das linhas estava correto, mostrando a mensagem "ok" e a contagem geral de caracteres estava no número correto de 60 caracteres, mostrando a mensagem final "ok"

** Generate an MNREAD sentence [computer program]. New York: Plattsburgh State University of New York, Department of Psychology; 2005. [cited 2005 Mar 19; last updated 2004 Dec 28]. Available from: http://faculty.plattsburgh.edu/steve.mansfield/ $\mathrm{CPR} /$ mnread 1 .asp 


\begin{tabular}{|c|c|c|c|c|c|c|c|c|}
\hline \multicolumn{9}{|c|}{ Palavras/minuto das 59 sentenças } \\
\hline Ordem & Frase & Média & Ordem & Frase & Média & Ordem & Frase & Média \\
\hline 1 & 38 & 209,8 & 21 & $13 b$ & 192,4 & 41 & 41 & 186,3 \\
\hline 2 & $5 a$ & 206,6 & 22 & 31 & 192,2 & 42 & $5 b$ & 186,2 \\
\hline 3 & 57 & 203,5 & 23 & 3a & 192,2 & 43 & $2 b$ & 185,9 \\
\hline 4 & $1 \mathrm{a}$ & 203,1 & 24 & $14 a$ & 192,0 & 44 & $1 b$ & 185,7 \\
\hline 5 & $6 a$ & 201,7 & 25 & 34 & 191,4 & 45 & 35 & 185,7 \\
\hline 6 & $12 a$ & 201,7 & 26 & $15 a$ & 191,0 & 46 & 46 & 185,4 \\
\hline 7 & $4 a$ & 197,9 & 27 & 52 & 190,5 & 47 & $10 a$ & 185,1 \\
\hline 8 & $6 b$ & 197,9 & 28 & $11 b$ & 190,5 & 48 & 49 & 184,9 \\
\hline 9 & $14 b$ & 197,3 & 29 & $9 b$ & 190,1 & 49 & $10 b$ & 183,0 \\
\hline 10 & 36 & 197,0 & 30 & 50 & 190,0 & 50 & 32 & 182,4 \\
\hline 11 & $8 a$ & 195,2 & 31 & 42 & 189,9 & 51 & 40 & 181,6 \\
\hline 12 & 55 & 195,2 & 32 & 45 & 189,8 & 52 & $11 a$ & 181,0 \\
\hline 13 & $7 a$ & 194,8 & 33 & 51 & 189,5 & 53 & $8 b$ & 180,2 \\
\hline 14 & $2 a$ & 194,1 & 34 & $13 a$ & 189,3 & 54 & $3 b$ & 179,4 \\
\hline 15 & 56 & 193,6 & 35 & 53 & 189,0 & 55 & $4 b$ & 178,8 \\
\hline 16 & 33 & 193,6 & 36 & 44 & 189,0 & 56 & 58 & 176,1 \\
\hline 17 & $12 b$ & 193,4 & 37 & 43 & 188,8 & 57 & $7 b$ & 175,8 \\
\hline 18 & $15 b$ & 193,1 & 38 & $9 a$ & 187,4 & 58 & 37 & 174,8 \\
\hline 19 & 54 & 192,8 & 39 & 59 & 186,5 & 59 & 47 & 171,3 \\
\hline 20 & 48 & 192,8 & 40 & 39 & 186,3 & & & \\
\hline
\end{tabular}

tes leram as 59 sentenças que estavam impressas em 3 linhas no centro, em folhas individuais. Foi utilizado a fonte Times New Roman, tamanho 24, para permitir uma leitura confortável e veloz. Foi também solicitado aos participantes que reportassem qualquer dificuldade de compreensão ou qualquer erro de construção da frase. As palavras lidas por minuto das sentenças, a média de velocidade e o desvio padrão foram calculados (Tabelas 1 e 2). O cálculo da velocidade de leitura foi realizado utilizando a fórmula de cálculo da MNREAD original. Foram descartadas as sentenças que foram lidas muito rapidamente e as sentenças que foram lidas muito lentamente.

Numa segunda fase, 70 novas frases foram elaboradas, totalizando 110. Análises de freqüência de palavras utilizadas em cada frase foram realizadas entre uma etapa e outra. A análise de freqüência de palavras foi realizada utilizando-se o aplicativo Perl*.

Esse aplicativo executado na plataforma Unix conta o número de ocorrência de cada palavra nas sentenças. A primeira análise foi realizada depois que a metade das sentenças foram escritas e uma lista de palavras foi feita para ser consultada para evitar que as novas frases utilizassem palavras repetidas. Algumas frases desta primeira etapa foram eliminadas, pois a freqüência de repetição estava acima do máximo permitido (duas vezes). A segunda análise foi realizada após a construção das 110 frases. Nesta fase, as sentenças passaram pela avaliação de uma professora de Português para a verificação de erros gramaticais. A terceira análise foi feita depois que as sentenças foram testadas nos adultos e a última análise foi realizada depois da seleção final das sentenças.

\footnotetext{
* Foundation Stone. Wordcount.pl [computer program]. Israel; 1998. [cited 2005 Mar 23]. Available from: http://foundationstone.com.au/HtmlSupport/WebPage/wordcount.html
}

\section{Seleção das frases}

Das 110 frases construídas, 38 foram selecionadas para compor a tabela MNREAD-P.

O processo de escolha das 38 sentenças que compõe a tabela passou por duas etapas: na primeira, as 110 sentenças foram lidas por adultos (experimento 1) e na segunda, as sentenças pré-selecionadas (experimento 2) foram lidas por crianças. Foram elaboradas 2 tabelas de acuidade (Protótipo 1 e Protótipo 2) com 19 sentenças cada uma. A ordem em que as 38 frases finais foram incluídas na tabela foi aleatória e os critérios de inclusão e exclusão das frases foram baseados na velocidade de leitura, erros cometidos durante a leitura, desvio padrão de velocidade e comentários dos examinados, como frase difícil ou estranha.

\section{Experimento 1 - Adultos}

Vinte adultos voluntários foram testados, 15 do sexo feminino e 5 do sexo masculino com idades variando de 22 a 47 anos. Quanto ao nível de escolaridade, 2 tinham o $2^{\circ}$ grau completo, 8 nível superior completo e 10 nível superior incompleto.

As frases numeradas foram colocadas em 4 diferentes seqüências escolhidas arbitrariamente e cada grupo de 5 participantes leu uma seqüência. O teste seguiu o protocolo descrito anteriormente no estudo piloto. Cada participante possuía um formulário com as frases impressas a serem lidas e foi anotado o tempo de leitura em segundos e as observações feitas por cada leitor. Todos os testes foram realizados pelo mesmo examinador.

Após a colheita dos dados, foi calculada a média de palavras por minuto de cada sentença e o desvio padrão das mesmas. A seleção das 69 sentenças foi realizada excluindo-se 


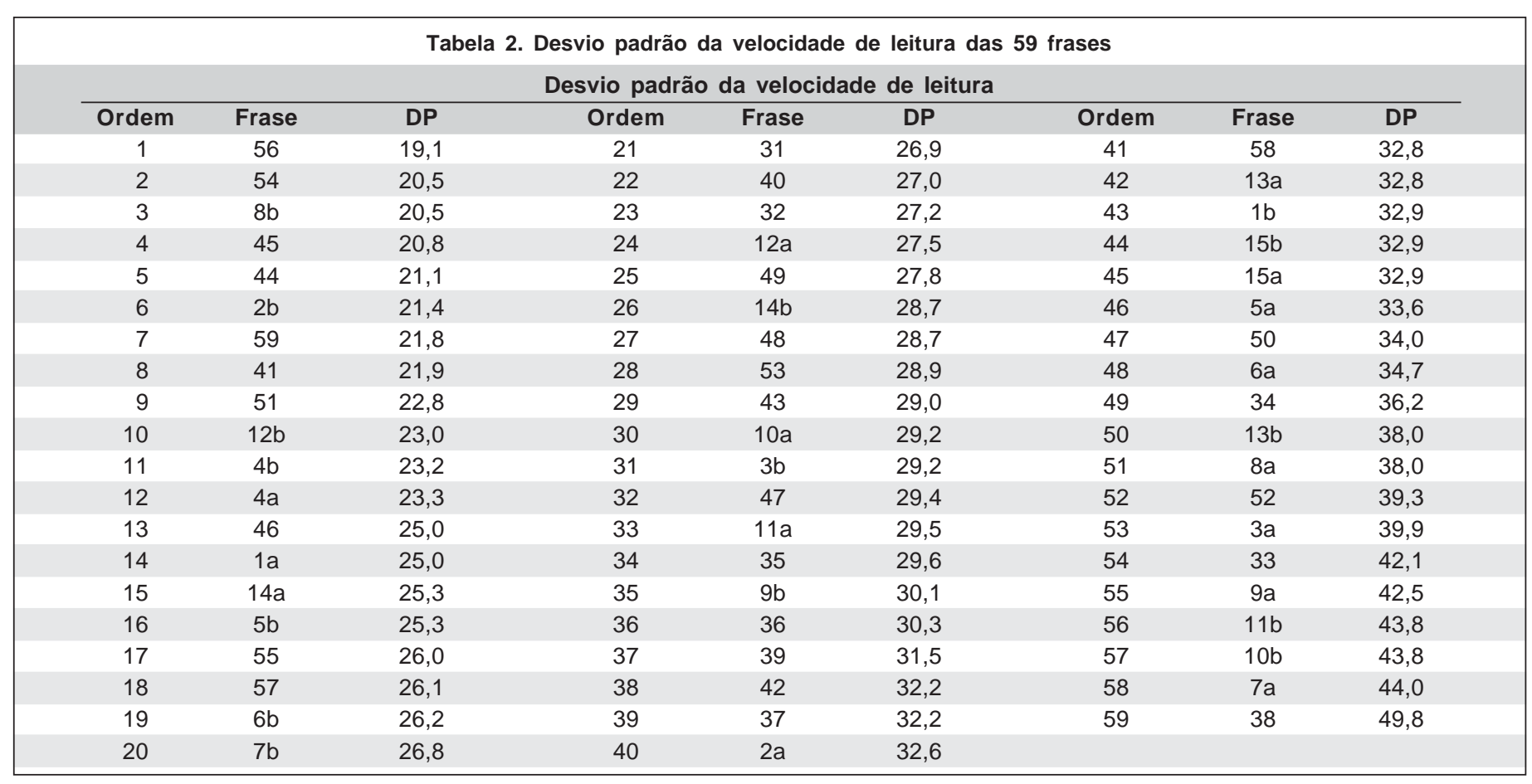

aquelas que possuíam média de velocidade de leitura rápida (acima de 196 palavras/min) e média de velocidade de leitura lenta (menos que 172 palavras/min). Também foram excluídas sentenças com desvios-padrão elevados (acima de 35,7) e também as sentenças que tiveram uma freqüência alta de comentários negativos (4 ou mais).

\section{Experimento 2 - Crianças}

Foram testadas 16 crianças da $3^{\underline{a}}$ série do ensino fundamental, todas com 9 anos de idade, 8 do sexo feminino e 8 do sexo masculino.

As 69 sentenças foram colocadas em 4 novas diferentes seqüências escolhidas arbitrariamente. Cada grupo de 4 crianças leu um conjunto diferente de frases. Os testes foram realizados obedecendo aos mesmos critérios utilizados no teste dos adultos, mas não foi solicitado que informassem os erros ou palavras que considerassem difíceis. Os erros cometidos foram anotados nas suas respectivas folhas de marcação.

A média da velocidade de leitura de cada frase e o desvio padrão foram calculados e as com velocidade muito lentas (menos que 95 palavras/min) ou muito rápidas (acima de 125 palavras/min) e frases que tinham grande desvio padrão (acima de 36) foram eliminadas. Outro critério adotado para a seleção das frases foi o da eliminação de sentenças que continham erros repetidos na leitura. Portanto, após estas seleções, as 38 melhores sentenças foram escolhidas para fazer parte das tabelas MNREAD-P.

\section{Elaboração da tabela MNREAD-P}

As 38 sentenças identificadas no processo de seleção foram separadas em 2 seqüências de 19 sentenças cada para compor as 2 tabelas MNREAD-P (Protótipo 1 e Protótipo 2). Cada sequiência não possuía frases com palavras iguais e não possuía frases com conteúdo semântico igual.

Após a elaboração dos 2 protótipos, as tabelas foram armazenadas em computador em arquivo PostScript e foram impressas numa impressora PostScript de alta resolução de 3600 pontos por polegada numa gráfica comercial.

\section{Validação da tabela MNREAD-P}

No processo de validação foram testados 20 adultos voluntários, sendo 10 do sexo masculino e 10 do sexo feminino. Os indivíduos foram testados na tabela MNREAD-P na distância de $40 \mathrm{~cm}$. Os indivíduos leram previamente um parágrafo de um texto $(0,6 \log \mathrm{MAR})$ representando um material de leitura do diaa-dia e a velocidade de leitura em palavras/minuto foi medida.

\section{RESULTADOS}

A velocidade de leitura dos 20 adultos na leitura das 110 frases variou de 140 palavras/minuto a 238 palavras/minuto e a velocidade de leitura das crianças na leitura das 69 frases variou de 63 palavras/minuto a 145 palavras/minuto.

O desempenho dos 20 adultos com AV normal no teste da tabela MNREAD-P é mostrado na figura 3. Cada ponto vermelho representa a velocidade de leitura de cada frase e o ponto em destaque mostra a velocidade de leitura realizada ao ler o parágrafo de texto previamente selecionado do estudo. Foi observado que a medida obtida da máxima velocidade de leitura da tabela MNREAD-P em adultos normais teve a média de 200,05 palavras/minuto e desvio padrão de 19,00 e não mostrou dife- 
rença significativa pelo teste $\mathrm{t}$-pareado de Student à máxima velocidade de leitura do parágrafo (média $=197,82$ palavras/ minuto e desvio padrão $=31,29$ ). Como mostrado na figura 4 , houve boa correlação com significância estatística entre estes dois parâmetros pelo teste de correlação de Pearson ( $\mathrm{r}=0,819$; $\mathrm{p}=0,607)$.

\section{DISCUSSÃO}

A confecção de uma tabela de medida de velocidade de leitura que tenha validade clínica, e que proporcione medidas precisas e replicáveis desta função, exige o cumprimento de vários quesitos. No presente estudo, foram observadas as regras de elaboração e seleção de frases pertinentes à construção da tabela MNREAD.
A acuidade e velocidade de leitura vêm sendo utilizadas como funções visuais em vários trabalhos envolvendo indivíduos de visão normal e visão baixa ${ }^{(15)}$. Foram utilizadas para comparar a velocidade de leitura no pré e pós-operatório de transplante de fotorreceptores de pacientes com retinose pigmentária ${ }^{(16)}$ e para comparar a melhora da função visual após a cirurgia de translocação macular em portadores de degeneração macular relacionada à idade ${ }^{(17)}$. O teste de velocidade de leitura mostrou-se útil para testar visão central em pacientes com catarata ${ }^{(18)}$, e também em estudo para comparar lentes intra-oculares bifocais, multifocais e monofocais ${ }^{(19)}$.

Nos pacientes com visão baixa, a acuidade e velocidade de leitura têm sido avaliadas no momento da escolha do auxílio óptico ideal ${ }^{(20)}$ e para determinar a magnificação ideal para os pacientes portadores de degeneração macular relacionada à idade $^{(21)}$. Foi sugerido que o indivíduo necessita de uma velo-

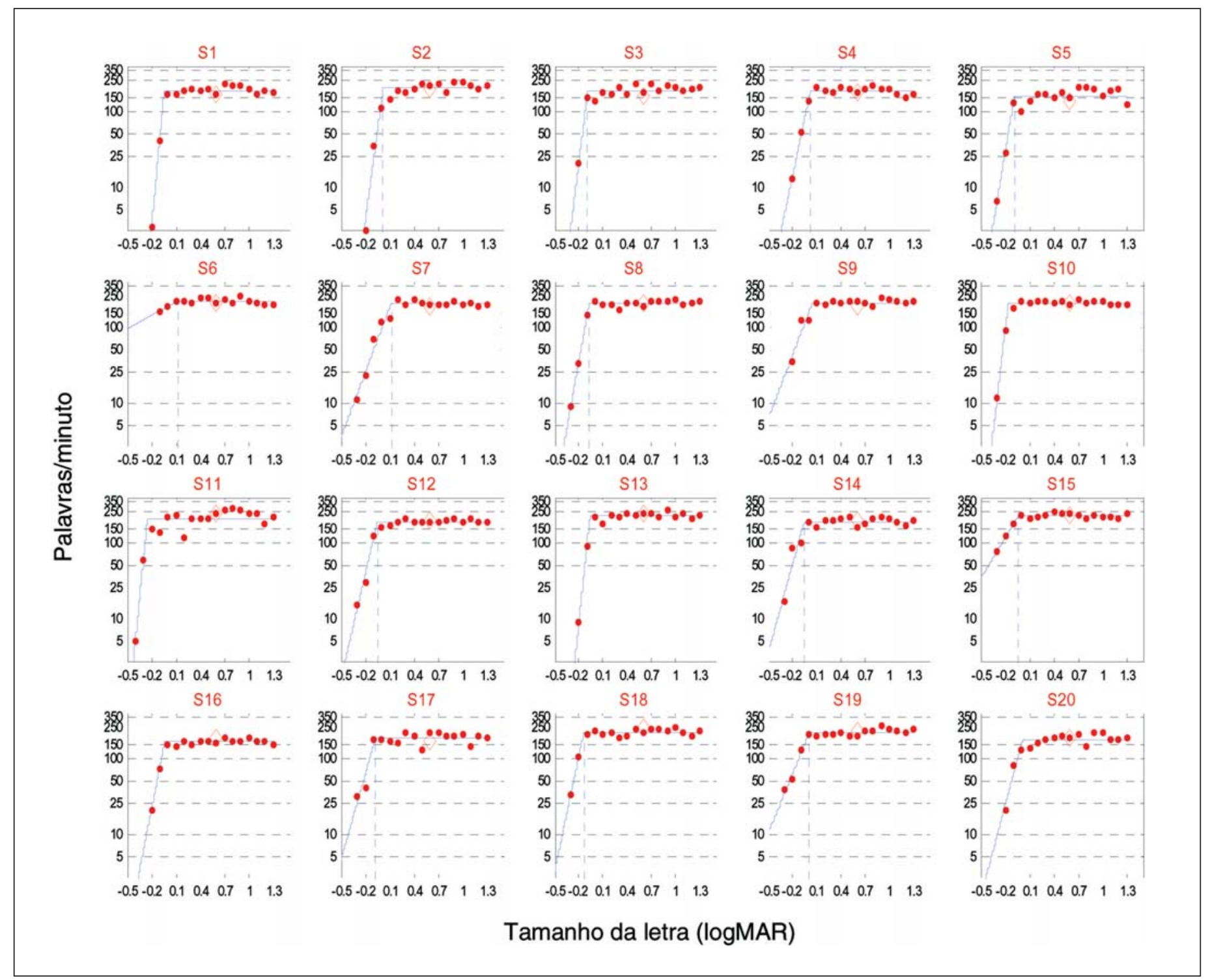

Figura 3 - Desempenho de velocidade de leitura de 20 adultos testados na tabela MNREAD-P e o desempenho de leitura do texto selecionado 


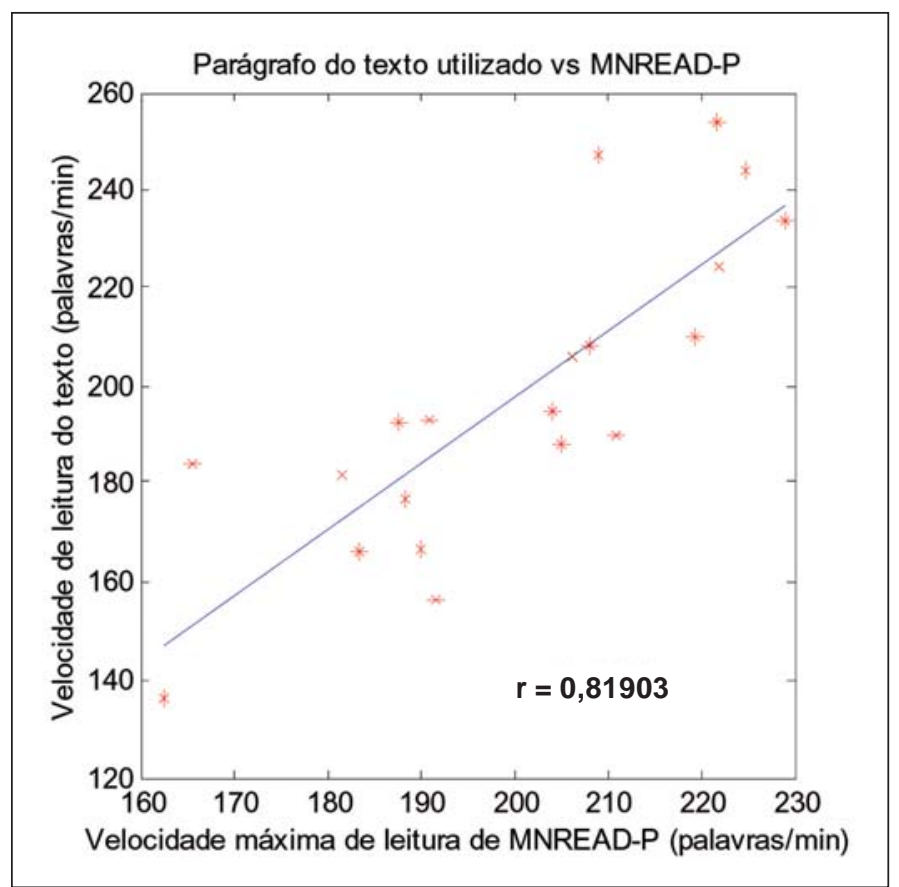

Figura 4 - Velocidade máxima de leitura da MNREAD-P (palavras/minuto) e do parágrafo do texto utilizado

cidade de leitura de 160 palavras/minuto para realizar uma leitura fluente e rápida, 80 palavras/minuto para realizar uma leitura fluente e 40 palavras/minuto para realizar leituras dirigidas em alguns pontos ${ }^{(22)}$.

Além da tabela MNREAD existem outras tabelas de velocidade de leitura, no entanto, a tabela MNREAD é a única que possui parâmetros psicofísicos padronizados que informam a acuidade de leitura, o tamanho crítico de letra, e a velocidade máxima de leitura. A acuidade de leitura é importante porque indica o menor tamanho de letra que o indivíduo consegue ler sem cometer erros. O tamanho crítico de letra é importante pois informa até que tamanho de letra o indivíduo consegue manter o seu potencial máximo de leitura e a velocidade máxima de leitura, que nos indica a velocidade de leitura quando a leitura não está limitada pelo tamanho da letra ${ }^{(23)}$.

Uma grande vantagem que a tabela MNREAD apresenta sobre as outras é o fato de poder utilizá-la em qualquer distância, pois utiliza o padrão logMAR de acuidade visual ${ }^{(24)}$.

De acordo com Oda, a mesma tabela de leitura pode ser utilizada repetidamente por 2 vezes consecutivas num intervalo de 2 semanas e ainda fornecer medidas confiáveis de velocidade de leitura ${ }^{(25)}$.

A tabela MNREAD na versão da língua portuguesa vem preencher uma lacuna do panorama nacional, já que não existe até o presente momento nenhum teste de função visual para perto padronizado e validado.

O uso da tabela MNREAD-P permitirá futuros estudos sobre os déficits apresentados por pacientes com visão baixa, além de ser um instrumento útil e validado para avaliar a eficácia dos auxílios ópticos de forma quantitativa. A MNREAD-P beneficiará além dos pacientes, a comunidade científica e profissionais da área oftalmológica.

\section{CONCLUSÕES}

Foram elaboradas e selecionadas 38 frases para a tabela MNREAD-P de acordo os princípios da tabela MNREAD original. A tabela MNREAD-P foi validada e aplicada em indivíduos normais e produziu o mesmo resultado que o MNREAD original. A velocidade de leitura medida na tabela MNREAD-P foi estatisticamente equivalente à medida de velocidade de leitura de um texto padrão de jornal.

\section{ABSTRACT}

Purpose: To create and to validate a version of the Minnesota Low Vision Reading Test (MNREAD) acuity chart for the Portuguese language. Methods: The Minnesota Low Vision Reading Test acuity chart contain 19 sentences $(\log$ MAR 0.5 to 1.3) with 60 characters printed on three lines. All the sentences must have the same length with simple vocabulary. A total of 110 sentences were generated. The sentences were presented to 36 subjects (20 adults and 16 children) and mistakes and reading time were marked. 38 sentences were selected for a prototype (MNREAD-P). Sentences with extreme high and low mean reading time, large standard deviation, and with persistent mistakes by subjects were excluded. Validation: Twenty subjects with normal vision ( $\log$ MAR 0 or 20/20 or better, with best refractive correction) were tested with the MNREAD-P and read a passage of text, representing normal, day-to-day reading. Reading speeds in words per minute were recorded for both the MNREAD and the text passage. Results: Sentences in the MNREAD Portuguese chart are sufficiently consistent to provide reliable measures of reading abilities. Reading speeds for the passage $(\log$ MAR $=0.6)$ were 197.8 words/minute and the maximum reading speeds calculated by the MNREAD-P were 200.1 words/minute. The correlation between the two measures was $r=0.82$. Conclusion: The MNREAD-P was tested on normal vision subjects and the results were the same from the original Minnesota Low Vision Reading Test. The reading speed measured on the MNREAD-P was statistically equivalent to the reading speed of the passage.

Keywords: Visual acuity; Vision; Reading; Vision, low/physiopathology; Vision tests; Psychophysics; Predictive value of tests; Software validation

\section{REFERÊNCIAS}

1. Legge GE, Ross JA, Isenberg LM, La May JM. Psychophysics of reading XII. Clinical predictors of low-vision reading speed. Invest Ophthalmol Vis Sci. 1992;33(3):677-87. 
2. Ross C, Stelmack JA, Stelmack TR, Fraim M. Preliminary examination of the reliability and relationship to clinical state of a measure of low vision patient functional status. Optom Vis Sci. 1990;68(12):919-23.

3. Legge GE, Klitz TS, Tjan BS. Mr Chips: an ideal-observer model of reading. Psychol Rev. 1997;104(3):524-53.

4. Sloan LL. Reading aids for the partially sighted: a systematic classification and procedure for prescribing. Baltimore: Williams \& Wilkins; 1977.

5. Bailey IL, Lovie JE. The design and use of a new near-vision chart. Am J Optom Opt. 1980;57(6):378-87.

6. Baldarase J, Watson GR, Whittaker SG, Miller-Shaffer HM - The development and evaluation of a reading test for low vision individuals with macular loss. J Vis Impair Blind. 1986;80:785-9.

7. Ahn SJ, Legge GE, Luebker A. Printed cards for measuring low-vision reading speed. Vision Res. 1995;35(13):1939-44.

8. Legge GE, Ross JA, Luebker A, LaMay JM. Psychophysics of reading VIII. The Minnesota Low-Vision Reading Test. Optom Vis Sci. 1989;66(12):843-53.

9. Watson GR, Wright V, De L'Aune W. The efficacy of comprehension training and reading practice for print readers with macular loss. J Visl Impair Blind. 1992;86(1):37-43.

10. Mansfield JS, Legge GE, Bane MC. Psychophysics of Reading XV. Font effects in normal and low vision. Invest Ophthalmol Vis Sci. 1996;37(8):1492-501.

11. Dietzch MJM. Um texto. Vários autores. Relações fala-escrita em textos de crianças das séries iniciais do primeiro grau [tese]. São Paulo: Instituto de Psicologia da Universidade de São Paulo; 1988.

12. Rodrigues AJ. Produção lingüística de crianças de seis anos: estudo comparativo do desempenho em situações e classes sociais diferentes [tese]. São Paulo: Instituto de Psicologia da Universidade de São Paulo; 1992.

13. Barrera SD. Linguagem oral e alfabetização: um estudo sobre variação lingüística e consciência metalingüística em crianças da $1^{\underline{a}}$ série do ensino fundamental [tese]. São Paulo: Instituto de Psicologia da Universidade de São Paulo; 2000.
14. Capovilla FC, Capovilla AGS. O desenvolvimento dos vocábulos receptivo e expressivo em crianças brasileiras. Rev Interamer Psicol. 1998;32(1)33-49.

15. Elliott DB, Patel B, Whitaker D. Development of a reading speed test for potential-vision measurements. Invest Ophthalmol Vis Sci. 2001;42(8):1945-9.

16. Berger AS, Tezel TH, Del Priore LV, Kaplan HJ. Photoreceptor transplantation in retinitis pigmentosa: short term follow-up. Ophthalmology. 2003;110 (2):383-91.

17. Fujikado T, Asonuma S, Ohji M, Kusaka S, Hayashi A, Ikuno Y, et al. Reading ability after macular translocation surgery with 360-degree retinotomy. Am J Ophthalmol. 2002;134(6):849-57.

18. Pesudovs K, Patel B, Bradbury JA, Elliott DB. Reading speed test for potential central vision measurement. Clin Experiment Ophthalmol. 2002; 30(3):183-6.

19. Richter-Mueksch S, Weghaupt H, Skorpik C, Velikay-Parel M, Radner W. Reading performances with a refractive multifocal and a diffractive bifocal intraocular lens. J Cataract Refract Surg. 2002;28(11):1957-63.

20. Peterson RC, Wolffsohn JS, Rubinstein M, Lowe J. Benefits of electronic vision enhancement systems (EVES) for the visually impaired. Am J Ophthalmol. 2003;136(6):1129-35

21. Cheong AMY, Lovie-Kitchin JE, Bowers AR. Determining magnification for reading with low vision. Clin Exp Optom. 2002;85(4):229-37.

22. Whittaker SG, Lovie-Kitchin J. Visual requirements for reading. Optom Vis Sci. 1993;70(1):54-65.

23. Legge GE, Ross JA, Maxwell KT, Luebker A. Psychophysics of reading VII Comprehension in normal and low vision. Clin Vision Sci. 1989;4(1):51-60.

24. Touzeau O. [Calculating the mean visual acuity and the change in visual acuity with decimal acuity chart]. J Fr Ophthalmol. 2003;26(6):586-90. French.

25. Oda K, Fujita C, Mansfield SJ, Legge GE. Does memory affect reading acuity measurement with MNREAD-J? In: Vision'99. International Conference of Low-vision. New York; 1999. 